\title{
CARACTERIZACIÓN FISICOQUÍMICA Y FITOQUÍMICA DE FRUTOS DE SAPUCHE (Randia laevigata Standl.)
}

\section{Physicochemical and phytochemical characterization of sapuche fruits (Randia laevigata Standl.)}

\author{
Luis Alfonso Jiménez Ortega ${ }^{1 *}$, Lucía Barrientos Ramírez ${ }^{2}$, Martín Pedro Tena Meza ${ }^{3}$
}

${ }^{1}$ Ciencia de los Alimentos, ${ }^{2}$ Departamento de Madera Celulosa y Papel, ${ }^{3}$ Departamento de Botánica y Zoología. Universidad de Guadalajara, Centro Universitario de Ciencias Biológicas y Agropecuarias, Lic. Camino Ramón Padilla Sánchez 2100, Nextipac, 44600 Zapopan, Jal, México.

* Autor para correspondencia: foodsciencetech@ hotmail.com

\section{Resumen}

El fruto de Randia laevigata Standl., es consumido por habitantes de la barranca del Río Santiago en el occidente de México. Diversas especies de Randia, son utilizadas en la medicina tradicional para el tratamiento de diferentes padecimientos, sin embargo es escasa la información química y biomédica de este género de plantas. El objetivo del presente trabajo fue caracterizar de manera preliminar los compuestos fitoquímicos y composición fisicoquímica de los frutos de $R$. laevigata Standl. Se liofilizaron los frutos maduros para la realización de los análisis fisicoquímicos: contenido de humedad, ceniza, proteína, extracto etéreo (grasa), fibra cruda, $\mathrm{pH}$ y grados Brix $\left({ }^{\circ} \mathrm{Bx}\right)$. Para el tamizaje fitoquímico se obtuvieron extractos con disolventes de diferente polaridad: hexano, diclorometano y metanol y se determinaron los tipos de metabolitos secundarios presentes en el fruto: fenoles, flavonoides, alcaloides, taninos, antraquinonas y saponinas. Dentro de la composición química proximal resalta la cantidad de proteína (3.31\%), extracto libre de nitrógeno $(35.75 \%)$, humedad $(57 \%)$, ceniza (3\%), extracto etéreo $(0.94 \%)$. Los metabolitos secundarios abundantes en el extracto metanólico fueron: saponinas, triterpenos, esteroides, compuestos fenólicos, antraquinonas y alcaloides, por lo que se sugiere continuar la investigación cuantificando y elucidando dichos compuestos, además de evaluarlos biológicamente.

Palabras clave: metabolitos secundarios, composición química proximal, productos naturales, fitoquímica.

\begin{abstract}
The Rio Santiago canyon residents in West Mexico consume Randia laevigata Standl fruit. A variety of species of the Randia is used in traditional medicine for treating some diseases. However, there is little information to be found on the diverse species of this plant. Therefore, the objective of this work is to determine the physicochemical and phytochemical compositions of Randia laevigata Standl fruit. Ripe fruit samples were lyophilized in order to carry out the physico-chemical analysis: humidity, ash, protein, ether extract, crude fiber, $\mathrm{pH}$ and degrees Brix $\left(^{\circ}\right.$ Bx). Extracts with solvents of different polarities were utilized during the phytochemical screening: hexane, dichloromethane and methanol. The analysis uncovered the most important families in the Sapuche: phenols, flavonoids, alkaloids,
\end{abstract}


tannins, quinones and saponins. The proximal chemical compositions of protein $(3.31 \%)$, nitrogen-free extract $(35.75 \%)$, humidity (57\%), ash (3\%), ethereal extract $(0.94 \%)$ stood out. Secondary metabolites, saponins, triterpenes, steroids and alkaloids, were found in abundance, suggesting that the research take a toxicological turn. Protein and nitrogen-free extract stood out in the nutritional analysis.

Key words: secondary metabolites, proximal composition, natural products, phytochemistry.

\section{Introducción}

Randia laevigata Standl. es un arbusto inerme de dos metros de alto que se distribuye desde los Estados Unidos de América hasta México, en este último, se localiza en los bosques tropicales caducifolios principalmente de los estados de Sonora, Durango, Nayarit, Jalisco, Michoacán, Guerrero, Oaxaca y Chiapas; presenta una distribución irregular al interior de la Barranca del Río Santiago. Posee hojas sésiles o con peciolos cortos, obovadas, oblongas, o rómbico-ovadas, que miden de 14 a $23 \mathrm{~cm}$ de largo; con ápice agudo o acuminado y base atenuada, con pubescencias cuando son jóvenes y glabras cuando han madurado (Standley, 1920); el fruto es una baya semi- globosa de $5-6.5 \mathrm{~cm}$ de largo y 3-4.3 cm de ancho, umbonada, glabra, con pericarpio coriáceo o leñoso; pedúnculo de 1.5-3.5 cm de largo (Figura 1); semillas numerosas comprimidas, elipsoidales a orbiculares de $8-10 \mathrm{~mm}$ de largo y de 6-8.5 $\mathrm{mm}$ de ancho, amarillentas o pardas, envueltas por una pulpa suculenta de color negro (Sahagún et al., 2014).

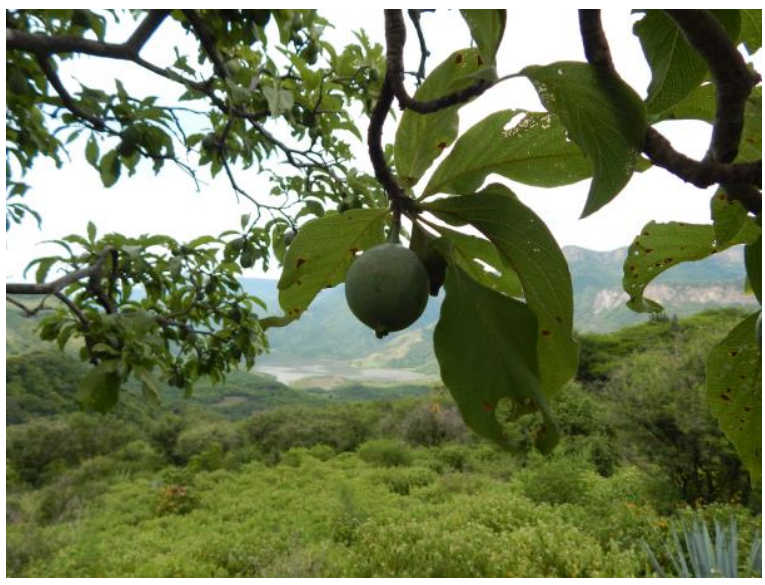

Figura 1: fruto de sapuche (Randia laevigata Standl).

El género Randia en México es utilizado como planta medicinal para tratar distintas enfermedades o padecimientos. Un ejemplo es la especie $R$. aculeata L., en Jamapa, Veracruz es usada para tratar mordeduras de serpiente (Gallardo et al., 2012); en el caso de $R$. uliginosa (Retz), se emplea para tratar enfermedades gastrointestinales y de vías urinarias (Sudhakar et al., 2012). R. monantha se utiliza en el caso de mordedura de serpientes $\mathrm{y}$ picaduras de animales ponzoñosos (Méndez y Hernández, 2009), y también contra problemas respiratorios (García, 2015). La pulpa de los frutos de $R$. echinocarpa DC., y $R$. capitata DC., se emplea en casi todo México destacando los estados de Michoacán y sus alrededores. Los usos frecuentes son para tratar padecimientos del sistema urinario, y del sistema respiratorio como la tos (Soto, 1987; Arizága, 2007). Se ha reportado el uso de Randia laevigata como remedio contra la bronquitis (Standley, 1920). También se han evaluado usos veterinarios por ejemplo, el extracto de $R$. armata ( $\mathrm{Sw}$ ), posee actividad contra la garrapata de los bovinos con un $75 \%$ de eficacia (Santos et al., 2013). Se han investigado las propiedades antiinflamatorias y antioxidantes de $R$. hebecarpa Benth., aislando cinco 
flavonoides, dos saponinas y un azúcar, atribuyendo a los flavonoides el efecto antioxidante de lípidos en especial del ácido linoleico con un $89.2 \%$ de inhibición casi igual que el butilhroxitolueno (BHT) con un $89.4 \%$ (Nazari et al., 2006). Irigoyen y Paredes, (2015) en su libro "Medicina Tarahumara: Etnobotánica y curación entre los rarámuri de México, nos hablan acerca del consumo de frutos de al menos tres especies de Randia: $R$. echinocarpa, $R$. watsonii y $R$. laevigata por parte de dicha comunidad indígena, donde se hace referencia que a pesar del sabor amargo, su consumo se consideraba como un manjar. Según los usos y actividades farmacológicas que se le dan a especies de Randia en México, se planteó estudiar los frutos de $R$. laevigata, los cuales carecen de información química que ayude a justificar los usos que se le atribuyen, por lo tanto el objetivo de la presente investigación fue caracterizar los frutos de Randia laevigata de manera fisicoquímica y fitoquímica, lo que podría contribuir a su conocimiento fitoquímico y etnofarmacológico.

\section{Materiales y métodos}

La investigación se realizó en el laboratorio de Química de Extraíbles ubicado en el Departamento de Madera Celulosa y Papel (DMCyP) y el laboratorio de Nutrición Animal, perteneciente al departamento de Salud Pública, de la Universidad de Guadalajara, en el Centro Universitario de Ciencias Biológicas y Agropecuarias, ubicado en Zapopan, Jalisco, México. El período de investigación abarcó desde la colecta de los frutos hasta el análisis de compuestos fisicoquímicos y fitoquímicos (diciembre, 2016-julio, 2018).

\section{Recolección y conservación de muestras}

Muestras de fruto maduro $(n=>30)$, se recolectaron de plantas ubicadas dentro del polígono ubicado en la parte noroeste del municipio de Amatitán, Jalisco, México, cuyos vértices se ubican de acuerdo con las siguientes coordenadas: $20^{\circ} 55^{\prime} 27^{\prime} ' \mathrm{~N}$ y $103^{\circ} 42^{\prime} 48^{\prime}$ 'O (vértice N.E); 2055'35'’N y $103^{\circ} 45^{\prime} 37^{\prime}$ 'O (vértice N.O); 20 $55^{\circ} 6^{\prime}$ ' $\mathrm{N}$ y $103^{\circ} 45^{\prime} 37^{\prime}$ 'O (vértice S.O); 2054'53' 'N y $103^{\circ} 42^{\prime}$ 56' 'W (vértice S.E). La recolección fue de manera manual, evitando dañar el fruto, se seleccionaron aquellos que no tuvieran daños físicos por plagas y de un tamaño de 6-7 cm de largo y de $4-5 \mathrm{~cm}$ de ancho. Para conservar las muestras sin que se degradarán los compuestos químicos $\mathrm{y}$ proceder a la realización de los análisis fisicoquímicos y fitoquímicos, se retiró manualmente el pericarpio y se homogenizó sólo el mesocarpio triturándolo en un mortero de porcelana, se congeló a $-16{ }^{\circ} \mathrm{C}$, esto para su posterior liofilización durante 72 horas en un sistema liofilizador marca Labconco Freeze $d r y{ }^{\circledR}$ modelo 4.5. Las variables de liofilización fueron: temperatura $-40{ }^{\circ} \mathrm{C}$ y una presión de 10 mbar. Las muestras liofilizadas se conservaron en tubos Falcon de $50 \mathrm{~mL}$ a una temperatura de -16 ${ }^{\circ} \mathrm{C}$. Cabe mencionar que para las determinaciones de humedad y cenizas se utilizó la pulpa sin liofilizar.

\section{Caracterización fisicoquímica}

Se realizaron análisis de humedad por el método secado en estufa, ceniza por el método de calcinación en seco (AOAC, 1990), proteína por digestión, extracto etéreo por el método Goldfisch, fibra cruda por digestión ácida y alcalina, $\mathrm{pH}$ y grados Brix $\left({ }^{\circ} \mathrm{Bx}\right)$, conforme las técnicas establecidas por la A.O.A.C. (Association of Official Analytical Chemists) (AOAC, 1990), todas las determinaciones se realizaron por duplicado. Posteriormente se realizó un cálculo de extracto libre de nitrógeno, utilizando los porcentajes de los componentes anteriores. 


\section{Caracterización fitoquímica}

Se realizó un tamizaje fitoquímico con los extractos hexánico (Hx), extracto de diclorometano (DCM) y extracto metanólico $(\mathrm{MeOH})$ obtenidos de los frutos. Con estos extractos, se determinaron: compuestos fenólicos, flavonoles, flavonas, flavonoides, alcaloides, antraquinonas, taninos, triterpenos, esteroides y saponinas de manera cualitativa mediante reacciones colorimétricas (Ardoino et al., 2013; Carvajal et al., 2009; Domínguez, 1973; Nath et al., 1946; Palacios, 2013; Robles et al., 2016).

\section{Resultados y discusión}

\section{Parámetros fisicoquímicos}

La composición química proximal de los frutos de $R$. laevigata se muestra en el cuadro 1 . Resalta su contenido de humedad $57 \%$ menor al $86.6 \%$ reportado para $R$. uliginosa y menor a $R$. laevigata (Nazarudeen, 2010), extracto libre de nitrógeno $35.75 \%, 12^{\circ} \mathrm{Bx}$ y un $\mathrm{pH}$ de 4.36 lo cual es menor que el $\mathrm{pH}$ de 5.51 para frutos de $R$. dumetorum reportado por
Kumar et al. (2010). Para el contenido de proteína, es bajo en comparación con hojas y tallos de $R$. aculeata la cual conforma la dieta del venado cola blanca en el ejido de Pitzotlán, Morelos, México, dicha especie aporta el $13.8 \%$ de proteína cruda (López et al., 2012). Los resultados obtenidos en cuanto a porcentaje de cenizas, son sólo mayores al de la especie $R$. uliginosa, sin embargo en ambos casos el contenido es mayor que otras frutas por ejemplo la guayaba, la yaca y la granada (Nazarudeen, 2010). Los frutos de la especie estudiada, contienen un porcentaje de grasa y fibra mínimo (Cuadro 1), considerado dentro de los parámetros reportados por el Sistema Mexicano de Equivalentes para la mayoría de las frutas y verduras (0-1\%) (Pérez et al., 2014). En el extracto libre de nitrógeno, pueden encontrarse algunos nutrientes importantes (Reveles et al., 2010; Saura et al., 1983). El contenido de grados Brix fue de $12{ }^{\circ} \mathrm{Bx}$, probablemente es la razón de que se le utilice en el proceso de fermentación del tesgüino, que es una bebida fermentada de maíz, llamada también Tejuino (Mudgil y Barak, 2018). En el Cuadro 2, se presenta una comparación.

Cuadro 1. Composición química proximal de frutos de Randia laevigata

\begin{tabular}{cc}
\hline Compuesto (\%) & \% en fruto \\
\hline Humedad & $57 \%$ \\
Cenizas & $3 \%$ \\
Proteína & $3.31 \%$ \\
Extracto etéreo & $0.94 \%$ \\
Fibra cruda & $0 \%$ \\
Extracto libre de nitrógeno & $35.75 \%$ \\
\hline
\end{tabular}


Cuadro 2. Comparación de la composición química proximal de diversas especies de Randia con R. Laevigata

\section{Especies de Randia}

\begin{tabular}{|c|c|c|c|c|c|c|}
\hline Análisis (\%) & $\begin{array}{l}\text { R. laevigata } \\
\text { (fruto) }\end{array}$ & $\begin{array}{l}\text { R. uliginosa } \\
\text { (fruto) }\end{array}$ & $\begin{array}{c}\text { R. echinocarpa } \\
\text { (hojas y tallos } \\
\text { frescos) }\end{array}$ & $\begin{array}{c}\text { R. aculeata } \\
\text { (hojas y } \\
\text { tallos) }\end{array}$ & $\begin{array}{l}\text { R. monantha } \\
\text { (frutos) }\end{array}$ & $\begin{array}{l}\text { R. dumetorum } \\
\text { (frutos) }\end{array}$ \\
\hline Humedad & $57 \%$ & $86.6 \%$ & N/D & N/D & $8.05 \% *$ & $78.3 \%$ \\
\hline Cenizas & $3 \%$ & $0.81 \%$ & $8.1 \%$ & $8.6 \%$ & N/D & $11.8 \%$ \\
\hline Proteína & $3.31 \%$ & $0.58 \%$ & $11.1 \%$ & $13.8 \%$ & $0.01 \% *$ & N/D \\
\hline Lípidos & $0.94 \%$ & $0.23 \%$ & N/D & N/D & N/D & N/D \\
\hline Fibra cruda & $0 \%$ & $2.88 \%$ & N/D & N/D & $1.26 \% *$ & N/D \\
\hline $\begin{array}{c}\text { Extracto } \\
\text { libre de } \\
\text { nitrógeno }\end{array}$ & $35.75 \%$ & N/D & N/D & N/D & N/D & N/D \\
\hline
\end{tabular}

Abreviaturas: $\mathrm{N} / \mathrm{D}=$ No disponible, $*$ determinación en base seca.

\section{Parámetros fitoquímicos}

Los resultados del tamizaje fitoquímico se presentan en los (Cuadros 3, 4 y 5). Para las muestras colectadas de $R$. laevigata, el extracto metanólico de los frutos, presentó mayor presencia de compuestos (Cuadro 5), esta abundancia de alcaloides detectada con esa fase del extracto, concuerda con lo señalado por Cruz et al., (2016) quienes concluyen que los principales metabolitos secundarios presentes en las hojas de $R$. nítida son alcaloides, compuestos fenólicos, taninos, flavonoides, cumarinas, triterpenos, y saponinas.
En diversos tamizajes fitoquímicos se revela la abundancia de alcaloides en hojas de distintas especies de Randia; resaltando en $R$. armata, $R$. diversiloba, $R$. karstenii, $R$. formosa y $R$. pepoformis y la presencia en $R$. aculeata, y $R$. lasiantha (Soto et al., 2001). Con base en estos resultados, se puede deducir que la abundancia de alcaloides y presencia de taninos son los responsables de conferir dicho sabor amargo a los frutos de esta especie, lo cual concuerda con lo reportado por Waizel y Waizel (2019). 
Cuadro 3. Tamizaje fitoquímico del extracto hexánico de frutos de Randia laevigata

\begin{tabular}{|c|c|c|c|c|c|c|}
\hline \multirow[b]{2}{*}{ Ensayos } & \multicolumn{6}{|c|}{ Metabolitos secundarios } \\
\hline & Fenólicos & Flavonoides & Alcaloides & Taninos & Antraquinonas & Saponinas \\
\hline Cloruro férrico & - & & & & & \\
\hline Constantinescu & & - & & & & \\
\hline Shinoda & & - & & & & \\
\hline Mayer & & & - & & & \\
\hline Dragendorff & & & - & & & \\
\hline Wagner & & & - & & & \\
\hline Hager & & & - & & & \\
\hline Gelatina-sal & & & & + & & \\
\hline Bornträger & & & & & - & \\
\hline Ac. afrógena & & & & & & + \\
\hline
\end{tabular}

Abundante (+++); presencia (+); dudoso (+/-); negativo (-)

Cuadro 4. Tamizaje fitoquímico del extracto de diclorometano de frutos de Randia laevigata

\begin{tabular}{|c|c|c|c|c|c|c|}
\hline \multirow[b]{2}{*}{ Ensayos } & \multicolumn{6}{|c|}{ Metabolitos secundarios } \\
\hline & Fenólicos & Flavonoides & Alcaloides & Taninos & Antraquinonas & Saponinas \\
\hline Cloruro férrico & - & & & & & \\
\hline Constantinescu & & - & & & & \\
\hline Shinoda & & - & & & & \\
\hline Mayer & & & - & & & \\
\hline Dragendorff & & & - & & & \\
\hline Wagner & & & - & & & \\
\hline Hager & & & - & & & \\
\hline Gelatina-sal & & & & - & & \\
\hline Bornträger & & & & & - & \\
\hline Ac. afrógena & & & & & & + \\
\hline
\end{tabular}

Abundante (+++); presencia (+); dudoso (+/-); negativo (-)

Cuadro 5. Tamizaje fitoquímico del extracto metanólico de frutos de Randia laevigata

\begin{tabular}{|c|c|c|c|c|c|c|}
\hline \multirow[b]{2}{*}{ Ensayos } & \multicolumn{6}{|c|}{ Metabolitos secundarios } \\
\hline & Fenólicos & Flavonoides & Alcaloides & Taninos & Antraquinonas & Saponinas \\
\hline Cloruro férrico & +++ & & & & & \\
\hline Constantinescu & & +++ & & & & \\
\hline Shinoda & & - & & & & \\
\hline Mayer & & & +++ & & & \\
\hline Dragendorff & & & +++ & & & \\
\hline Wagner & & & +++ & & & \\
\hline Hager & & & +++ & & & \\
\hline Gelatina-sal & & & & - & & \\
\hline Bornträger & & & & & +++ & \\
\hline Ac. afrógena & & & & & & +++ \\
\hline
\end{tabular}

Abundante (+++); presencia (+); dudoso (+/-); negativo (-) 
El extracto hexánico y de diclorometano de frutos de $R$. laevigata carece de flavonoides, en contraste con el extracto metanólico. Esto concuerda con los resultados reportados por Cruz et al., (2016) y Santos et al. (2007), quienes señalan la presencia de dichos compuestos en el extracto metanólico de hojas de $R$. nítida el cual contiene una alta presencia de fenoles totales con $524.52 \pm 0.46 \mathrm{mg}$ equivalentes de ácido gálico y flavonoides con $178.47 \pm 0.98$ mg equivalentes de quercetina. Sin embargo también reportó que en el extracto hexánico había presencia de triterpenos y esteroides coincidiendo con los resultados de este trabajo. En el extracto de diclorometano no se encontró la presencia de compuestos fenólicos, cumarinas y alcaloides, mientras que los autores mencionados los reportan como presentes.

El extracto hexánico y de diclorometano de $R$. laevigata arrojó presencia de saponinas, mientras que el extracto metanólico abundancia. Estos resultados sugieren que se continúe la determinación de estos compuestos por otros métodos más precisos y que puedan detectar a nivel de derivados del ácido ursólico y oleanólico ( $\alpha$ y $\beta$-amirina), tal como señalan los resultados reportados por Bye et al. (1991). La placa cromatografíca con el reactivo Liebermann-Burchard indicó la presencia cualitativa de triterpenos y esteroles concordando con lo señalado por autores que reportan la presencia de estos compuestos en otras especies del mismo género (Carvajal et al., 2009; Santos et al., 2007)

\section{Conclusiones}

La presente investigación es el primer reporte de caracterización fisicoquímica y fitoquímica de los frutos del sapuche. Los resultados obtenidos demuestran el alto contenido de minerales en los frutos maduros de plantas de esta especie. Debido a la alta y dispersa presencia de alcaloides en especies de Randia, incluyendo $R$. laevigata, se recomienda aislarlos, elucidarlos y posteriormente evaluarlos biológicamente ya que se ha demostrado su actividad antibacteriana. La abundancia de compuestos fenólicos como los flavonoides presentes en el fruto puede justificar los usos etnofarmacológicos que se le atribuyen, sin embargo la investigación tuvo limitantes en cuanto a identificar, cuantificar y evaluar los compuestos antes mencionados por lo que se recomienda tener precaución en su consumo, además de que no se han realizado estudios donde se compruebe científicamente los beneficios a la salud a partir de su consumo. Es importante la investigación de plantas y frutos usados medicinalmente por comunidades indígenas de México, debido a que en la mayoría de las ocasiones es empírico el conocimiento que se tiene acerca de ellas.

\section{Literatura citada}

AOAC. (1990). Association of Official Analytical Chemistry. Official Method of Analysis. Virginia, USA: 16a. Edición.

Ardoino, S.M., M.A. Boeris y R.E. Toso. (2013). Caracterización fitoquímica de Prosopis flexuosa var. flexuosa (algarrobo) y Prosopis flexuosa var. depressa (alpataco), plantas con acción farmacológica*. Revista Ciencias Veterinarias, 15(1):115125.

HTTP://DX.DOI.ORG/10.19137/CIENVET 2013-15110.

Arizága, S. (2007). Estudio y colección viva de plantas medicinales nativas $\mathrm{y}$ formación de un banco de germoplasma del estado de Michoacán. 66 pp. [Publicación en 
línea]. Disponible desde internet en: <http://www.ine.gob.mx/dgioce/con_e co/informes2007.html>. [Con acceso el 12 febrero 2019, 12:45].

Bye, R., E. Linares., R. Mata., C. Albor., P.C. Castañeda y G. Delgado. (1991). Ethobotanical and phytochemical investigation of Randia echinocarpa (Rubiaceae). Anales del instituto de biología serie botánica, 62(1): 87-106.

Carvajal, R.L., Y.U. Hata., N.M. Sierra y N.D. Rueda. (2009). Análisis fitoquímico preliminar de hojas, tallos y semillas de cupatá (Strychnos schultesiana Krukoff). Revista Colombia Forestal, 12(1):161-170. https://doi.org/10.14483/udistrital.jour. colomb.for.2009.1.a11.

Cruz, S.S.C.B.D., R Matias, M.J.A. Bono, S.K. Silva y J. Ludwig. (2016) Antifungal potential of extracts and fractions of Randia nitida leaves on soybean pathogens and their phytochemistry. Revista caatinga, 29(3):594-602.

https://doi.org/10.1590/1983-

21252016v29n309rc.

Domínguez, X.A. (1973). Métodos de investigación fitoquímica. Limusa, Ciudad de México, México. 281 pp. [ISBN: 9681801156 9789681801151].

Gallardo, C.C.A, B.G. Guevara., R.E. Morales., J.Y. Tadeo., F.O. Gutiérrez., S.N. Jiménez., M.T.O. Valadez., V.M.T. Valenzuela y H.M.C. Castillo. (2012). Ethnobotanic study of Randia aculeata (Rubiaceae) in Jamapa, Veracruz, Mexico, and its anti-snake venom effects on mouse tissue. Journal of Venomous Animals and Toxins including Tropical
Diseases, 18(3), $287-$ 294. https://doi.org/10.1590/S167891992012000300006.

García, A.I.B. (2015). Plantas Medicinales, usos y costumbres: Estudio de Caso en dos Comunidades del Municipio de Puente Nacional, Veracruz. Tesis Ing. Agrónomo. Universidad Autónoma Agraria Antonio Narro. [Publicación en línea]. Disponible desde Internet en <http://repositorio.uaaan.mx:8080/xml ui/bitstream/handle/123456789/6841/ T20517\%20GARCIA\%20BARRADAS\%2c\% 20ANGEL\%20IVAN\%20\%2063433.pdf?s equence $=1$ \&isAllowed $=\mathrm{y}>$. [Consultado 16 Marzo 2020, 10:23].

Irigoyen, R.F y A. Paredes. (2015). Tarahumara medicine: ethnobotany and healing among the Rarámuri of Mexico. University of Oklahoma Press. Oklahoma, United States of America. 383 pp. ISBN: 9780806143620 .

Kumar, M.A., A. Singh., M.M. Rao y S. Kumari. (2010). Evaluation of preliminary phytochemical and physicochemical studies on the fruit of Randia dumetorum Lam. Asian Journal of Traditional Medicines, 5(2):75-78.

López, P.E., A.N. Serrano., V.B.C. Aguilar y C.A. Herrera. (2012). Composición nutricional de la dieta del venado cola blanca (Odocoileous virginianus ssp. mexicanus) en Pitzotlán, Morelos. Revista Chapingo serie ciencias forestales y del ambiente, 18(2):219-

229. https://dx.doi.org/10.5154/r.rchsc fa2011.01.006.

Méndez, V.L.M. y M.M.R. Hernández. (2009). Evaluación de la toxicidad del fruto de Randia monantha Benth. 
Revista Médica UV, Suplemento 1, 9(1):42-45.

Nath, M., M. Chakravorty y S. Chowdhury. (1946). Liebermann-Burchard Reaction for Steroids. Nature 157, 103-104. https://doi.org/10.1038/157103b0.

Nazari, A.S., S.A. Dias, W.F. da Costa, A.C.A. Bersani., G.J. Vidotti, M.C. de Souza y M.H. Sarragiotto. (2006). Anti-inflammatory and Antioxidant Activities of Randia hebecarpa and Major Constituents. Pharmaceutical Biology, 44(1): 7-9. https://doi.org/10.1080/138802005004 96504.

Nazarudeen, A. (2010). Nutritional composition of some lesser-known fruits used by the ethnic communities and local folks of Kerala. Indian Journal of Traditional Knowledge, 9(2): 398-402.

Palacios, P.M.I. (2013). Universidad Católica Los Ángeles de Chimbote, Texto digital de farmacognosia $y$ fitoquímica. 164 pp. [Publicación en línea]. Disponible desde Internet en <https://www.academia.edu/5271729/ UNIVERSIDAD_CAT\%C3\%93LICA_LOS_A NGELES_DE_CHIMBOTE_FACULTAD_DE _CIENCIAS_DE_LA_SALUD_ESCUELA_PR OFESIONAL_DE_FARMCIA_Y_BIOQU\%C 3\%8DMICA_TEXTO_DIGITAL_DE_FARM ACOGNOSIA_Y_FITOQU\%C3\%8DMICA>. [Consultado 06 Abril 2020, 10:23].

Pérez, L.A.B., G.B. Palacios y A.L.B. Castro. (2014). SMAE, Sistema mexicano de alimentos equivalentes. Fomento de nutrición y salud. Ciudad de México, México. 164 pp. [ISBN: 9786070079283].

Reveles, S.F.O., S.R. Rosales., B.C.A. Nava., L.E. Delgado., R.E.I. Cuéllar., C.F.O. Carrete y S.J.C. Ríos. (2010).
Identificación de especies vegetales con potencial para la producción de biocombustibles líquidos en Durango, México. Revista Mexicana de Ciencias Agrícolas, 1(1):45-54.

Robles, G.M.A., A.J. Aguilar., L.M. Gutiérrez., Rodríguez, F.F., J.A. Morales., M.P.J. Guerrero., P.J.A. Madrigal y S.C.L. Del Toro. (2016). Identificación cualitativa de metabolitos secundarios y determinación de la citotoxicidad de extractos de tempisque (Sideroxylum capiri Pittier). Biotecnia, 18(3):38. http://dx.doi.org/10.18633/biotecnia .v18i3.328.

Sahagún, G. E., M.A.R Macías., P.R. Carrillo., N.G Larrañaga y J.A.G Vázquez. (2014). Guía de campo de los árboles tropicales de la Barranca del Río Santiago en Jalisco, México. Universidad Autónoma de Guadalajara, México. 315 pp. ISBN 607833624X, 9786078336241.

Santos, C.M.E., Z.M.E. Zazueta., P.G. Loarca., L.O. Paredes y V.F. Delgado. (2007). Antioxidant and Antimutagenic Activities of Randia echinocarpa Fruit. Plant Foods for Human Nutrition, 62:71-77. https://doi.org/10.1007/s11130-0070044-x.

Santos, L.B., J.K. Souza., B. Papassoni., B.D.G. Lino., J.G.A. Damasceno., J.M. Escher de Souza., C.A. Carollo y B.F. Almeida. (2013). Efficacy of extracts from plants of the Brazilian Pantanal against Rhipicephalus (Boophilus) microplus. Revista Brasileira de Parasitologia Veterinária, 22(4), 532538. https://doi.org/10.1590/S198429612013000400013 
Saura, C.F., J. Cañellas y L. Soler. (1983). Dietary Fibre and Components of the Nitrogen-free Extract of Almond Kernels. Journal of the Science of Food and Agriculture, 34(12):14191422.

https://doi.org/10.1002/jsfa.27403412

16.

Soto, J. (1987). Las plantas medicinales y su uso tradicional en la Cuenca del Río Balsas; Estados de Michoacán y Guerrero, México. Tesis. Lic. En Biología. Universidad Nacional Autónoma de México. [publicación en línea]. Disponible desde Internet en

<http://132.248.9.195/pmig2018/0045 116/0045116.pdf >. [Consultado 16 Marzo 2020, 10:23].

Soto, S.A., B. Castillo, A. Delgado, A. González y R. Montenegro. (2001). Alkaloid screening of herbarium samples of Rubiaceae from Panama. Pharmaceutical Biology, 39(3): 131169.

https://doi.org/10.1076/phbi.39.3.161.5 925.

Standley, C.P. 1920. United States National Herbarium, volume 23, Trees and shrubs of Mexico. US Government Printing Office, Washington, United States of America. $1721 \mathrm{pp}$. https://doi.org/10.5962/bhl.title.15726.

Sudhakar, K., M.C. Eswaraiah., M.M. Eswarudu., K.P. Kumar y K. Nagaraju. (2012). An updated review on Tamilnadia uliginosa. International Research Journal of Pharmaceutical and Applied Sciences, 2(5):185-189. ISSN: 22774149.

Waizel, B.J y H.S. Waizel. (2019). Las plantas con principios amargos y su uso medicinal. ¿Un futuro dulce?
Anales de Otorrinolaringología Mexicana, 64(4):202-228. 